\title{
Modified Nonradial Supper Efficiency Models
}

\author{
H. Jahanshahloo, ${ }^{1}$ F. Hosseinzadeh Lotfi, ${ }^{1}$ and M. Khodabakhshi ${ }^{2}$ \\ ${ }^{1}$ Department of Mathematics, Science and Research Branch, Islamic Azad University, Tehran, Iran \\ ${ }^{2}$ Department of Mathematics, Faculty of Mathematical Sciences, Shahid Beheshti University, G.C., Tehran, Iran
}

Correspondence should be addressed to H. Jahanshahloo; hoda@jahanshahloo.com

Received 25 November 2013; Accepted 28 December 2013; Published 17 February 2014

Academic Editor: S. H. Nasseri

Copyright (C) $2014 \mathrm{H}$. Jahanshahloo et al. This is an open access article distributed under the Creative Commons Attribution License, which permits unrestricted use, distribution, and reproduction in any medium, provided the original work is properly cited.

Ranking Efficient Decision Making Units (DMUs) are an important issue in Data Envelopment Analysis (DEA). This is one of the main areas for the researcher. Different methods for this purpose have been suggested. Appearing nonzero slack in optimal solution makes the method problematic. In this paper, we modify the nonradial supper efficiency model to remove this difficulty. Some numerical examples are solved by modified model.

\section{Introduction}

Data Envelopment Analysis is a mathematical programming technique which evaluates the relative efficiency of DMUs. DEA classifies the DMUs into two different classes, called set of efficient DMUs and the set of inefficient DMUs.

Conventional DEA model cannot differentiate the efficient DMUs whose efficiency value is one. Toward this end, different methods are suggested; see [1-3]. One of the important models is AP-Model which was proposed by Andersen and Petersen [4] and also see [5, 6].

This model is widely used and the results are almost satisfactory. The main deficiencies of AP model are being as follows:

(1) infeasible for some kind of data,

(2) unstable in the sense that a small variation in data causes big increase (degrease) in the result,

(3) not taking into account the nonzero slacks which appear in optional solution (projection of omitted DMU is weak efficient in new PPS).

For removing these difficulties, many researches have suggested different models; for example, see [7-9].

Tone [7] suggested the nonradial supper efficiency model. This model fails to remove the 3 rd deficiency. In this paper, we modify the nonradial supper efficiency model that takes into account nonzero slack that appears in optimal solution.
The rest of the paper is organized as follows. In Sections 2 and 3, AP-model and Tone's model are discussed. Section 4 contains the modified model. In Sections 5 and 6, input and output oriented models are proposed. Discussion and conclusion cover Section 7.

\section{Anderson Peterson (AP) Model}

Consider $n$ decision making units $\operatorname{DMU}_{j}(j=1, \ldots, n)$ which consumes $0 \neq x_{j} \geq 0$ and $x_{j} \in R^{m}(j=1, \ldots, n)$ vector as input to produce output vector $0 \neq y_{j} \geq 0$ and $y_{j} \in R^{s}(j=1, \ldots, n)$. The supper efficiency model may be written as follows:

$$
\begin{aligned}
& \theta_{p}^{*}=\operatorname{Min} \theta \\
& \text { s.t. } \sum_{j=1, j \neq p}^{n} \lambda_{j} x_{i j}+s_{i}^{-}=\theta x_{i p} \quad i=1, \ldots, m \\
& \quad \sum_{j=1, j \neq p}^{n} \lambda_{j} y_{r j}-s_{r}^{+}=y_{r p} \quad r=1, \ldots, s \\
& \lambda_{j} \geq 0 \quad j=1, \ldots, n, j \neq p \\
& s_{i}^{-} \geq 0 \quad i=1, \ldots, m \\
& s_{r}^{+} \geq 0 \quad r=1, \ldots, s .
\end{aligned}
$$




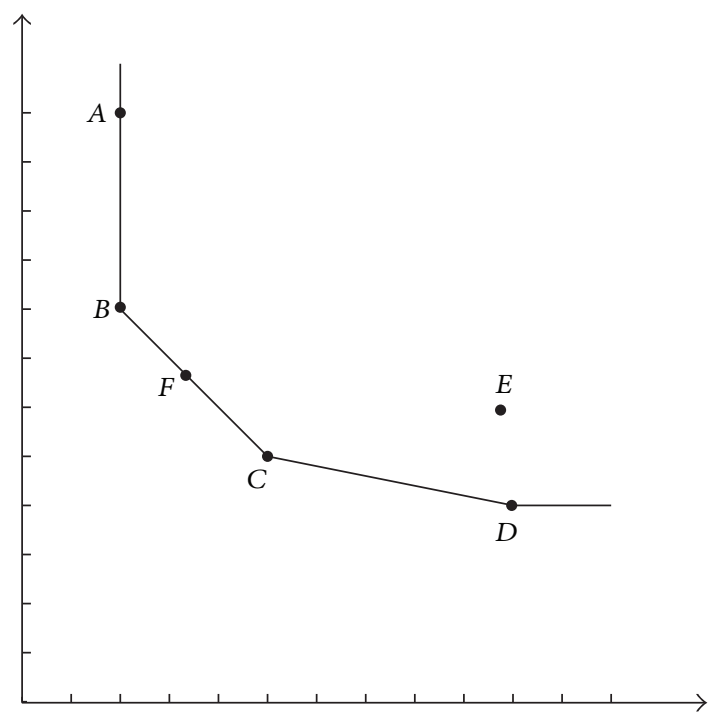

FIGURE 1

The following example which has been taken from [7] shows the deficiency in case of having nonzero slacks in optimal solution.

Example 1. Consider the data given by Table 1.

By using AP-Model,

$$
\begin{aligned}
& \text { Sup } A=\theta_{A}^{*}=1 \quad S_{2}^{*}=4 \\
& \text { Sup } B=\theta_{B}^{*}=1.260 \quad \text { All slacks are zero } \\
& \text { Sup } C=\theta_{C}^{*}=1.133 \text { All slacks are zero } \\
& \text { Sup } D=\theta_{D}^{*}=1.250 \quad S_{1}^{-*}=7.5 \\
& \text { Sup } E=\theta_{E}^{*}=0.750 \\
& \text { Sup } F=\theta_{F}^{*}=1 \text {. }
\end{aligned}
$$

In other words, the supper efficiencies are as follows.

$$
\begin{aligned}
\theta_{A}^{*} & =1-4 \varepsilon(*) \\
\theta_{B}^{*} & =1.260 \\
\theta_{C}^{*} & =1.133 \\
\theta_{D}^{*} & =1.25-7.5 \varepsilon(*) \\
\theta_{E}^{*} & =0.750 \\
\theta_{F}^{*} & =1(*) .
\end{aligned}
$$

Note. These values are not correct in [7], because the result shown in the paper has taken from original paper in which $F$ is not included. From Figure 1 it can be seen that the radial supper Efficiency model is not able to rank $F$ which is nonextreme efficient and also is not able to rank $D$ and $A$, with nonzero slack in optimal solutions.
TABLE 1

\begin{tabular}{lcccccc}
\hline DMU & $A$ & $B$ & $C$ & $D$ & $E$ & $F$ \\
\hline Input 1 & 2 & 2 & 5 & 10 & 10 & 3.5 \\
Input 2 & 12 & 8 & 5 & 4 & 6 & 6.5 \\
Output 1 & 1 & 1 & 1 & 1 & 1 & 1 \\
\hline
\end{tabular}

TABLE 2

\begin{tabular}{lcccccccc}
\hline DMU & $A$ & $B$ & $C$ & $D$ & $E$ & $F$ & $G$ & $H$ \\
\hline Input 1 & 4 & 7 & 8 & 4 & 2 & 10 & 12 & 10 \\
Input 2 & 3 & 3 & 1 & 2 & 4 & 1 & 1 & 1.5 \\
Output 1 & 1 & 1 & 1 & 1 & 1 & 1 & 1 & 1 \\
\hline
\end{tabular}

\section{Nonradial Supper Efficiency Model (Tone's Model)}

In 2002, Tone proposed the following nonradial supper efficiency model:

$$
\begin{aligned}
& \rho_{p}^{*}=\operatorname{Min} \frac{(1 / m) \sum_{i=1}^{m}\left(\bar{x}_{i} / x_{i p}\right)}{(1 / s) \sum_{r=1}^{s}\left(\bar{y}_{r} / y_{r p}\right)} \\
& \text { s.t. } \sum_{j=1, j \neq p}^{n} \lambda_{j} x_{i j} \leq \bar{x}_{i} \quad i=1, \ldots, m \\
& \sum_{j=1, j \neq p}^{n} \lambda_{j} y_{r j} \geq \bar{y}_{r} \quad r=1, \ldots, s \\
& \bar{x}_{i} \geq x_{i p} \quad i=1, \ldots, m \\
& \bar{y}_{r} \leq y_{r p} \quad r=1, \ldots, s \\
& \bar{y}_{r} \geq 0 \quad r=1, \ldots, s \\
& \lambda_{j} \geq 0 \quad j=1, \ldots, n, j \neq p .
\end{aligned}
$$

Based on the SBM model (4), the nonradial supper efficiency model should be as follows:

$$
\begin{aligned}
& \gamma_{p}^{*}=\operatorname{Min} \frac{1+(1 / m) \sum_{i=1}^{m}\left(s_{i}^{+} / x_{i p}\right)}{1-(1 / s) \sum_{r=1}^{s}\left(s_{r}^{-} / y_{r p}\right)} \\
& \text { s.t. } \sum_{j=1, j \neq p}^{n} \lambda_{j} x_{i j}-s_{i}^{+}=x_{i p} \quad i=1, \ldots, m \\
& \sum_{j=1, j \neq p}^{n} \lambda_{j} y_{r j}+s_{r}^{-}=y_{r p} \quad r=1, \ldots, s \\
& \lambda_{j} \geq 0 \quad j=1, \ldots, n, j \neq p \\
& s_{i}^{+} \geq 0 \quad i=1, \ldots, m \\
& s_{r}^{-} \geq 0 \quad r=1, \ldots, s .
\end{aligned}
$$

We will show that (4) and (5) are not equivalent, in the sense that their optimal solutions are different.

Example 2. Consider the eight Decision Making Units (DMU), with two inputs and one output (see Table 2). 


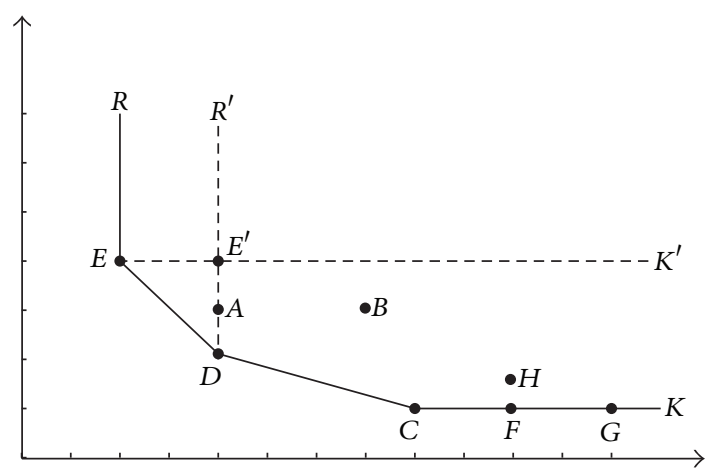

FIgURE 2

First the following are defined:

$$
\begin{gathered}
P P S=\left\{(x, y) \mid x \geq \sum_{j=1}^{n} \lambda_{j} x_{j},\right. \\
\left.y \leq \sum_{j=1}^{n} \lambda_{j} y_{j}, \lambda_{j} \geq 0, j=1, \ldots, n\right\}, \\
\overline{P P S}=\left\{(x, y) \mid x \geq \sum_{j=1, j \neq p}^{n} \lambda_{j} x_{j},\right. \\
\left.y \leq \sum_{j=1, j \neq p}^{n} \lambda_{j} y_{j}, \lambda_{j} \geq 0, j=1, \ldots, n\right\}, \\
\overline{\overline{P P S}}=\overline{P P S} \cap\left\{(x, y) x \geq x_{p}, y \leq y_{p}\right\} .
\end{gathered}
$$

In above-mentioned example and shown in Figure 2,

$$
\begin{gathered}
P P S=R E D C K=S \\
\overline{P P S}=R^{\prime} E^{\prime} D C K=\bar{S} \\
\overline{\overline{R^{\prime} E^{\prime} K^{\prime}}}=\overline{\bar{S}} .
\end{gathered}
$$

We can see that $\overline{\bar{S}}$ is a proper subset of $\bar{S}$ and $\bar{S}$ is a proper subset of $S$; that is,

$$
\overline{\bar{S}} \subset \bar{S} \subset S \text {. }
$$

In this example, the optimum value of objective function in (4) is

$$
\rho_{E}^{*}=\frac{1}{2}\left(\frac{4}{2}+\frac{4}{4}\right)=1.5
$$

and the optimal value of objective function in (5) is

$$
\gamma_{\bar{S}}^{*}=\frac{1}{2}\left(\frac{4}{2}+\frac{2}{4}\right)=\frac{1}{2}\left(2+\frac{1}{2}\right)=\frac{5}{4}=1.25,
$$

so (4) and (5) are not equivalent. In other words, the constraints $\bar{x} \geq x_{p}$ and $\bar{y} \leq y_{p}$ and $\bar{y} \geq 0$ should be omitted from (4). Now we show that the nonradial supper efficiency model has the same difficulty as radial supper efficiency model in treating nonzero slacks in optimal solution. Using
(5), the supper efficiency of $E, D, C$ may be evaluated as follows:

$$
\begin{array}{ll}
\gamma_{E}^{*}=\operatorname{Min} \quad & \left(1+\frac{1}{2} \sum_{i=1}^{2} \frac{s_{i}^{+}}{x_{i E}}\right) \\
\text { s.t. } \quad & 4 \lambda_{A}+7 \lambda_{B}+8 \lambda_{C}+4 \lambda_{D}+10 \lambda_{F}+12 \lambda_{G} \\
& +10 \lambda_{H}-s_{1}^{+}=2 \\
& 3 \lambda_{A}+3 \lambda_{B}+\lambda_{C}+2 \lambda_{D}+\lambda_{F}+\lambda_{G}+1.5 \lambda_{H} \\
& -s_{2}^{+}=4 \\
& \lambda_{A}+\lambda_{B}+\lambda_{C}+\lambda_{D}+\lambda_{F}+\lambda_{G}+\lambda_{H}+s^{-}=1 \\
& \lambda_{A}, \lambda_{B}, \lambda_{C}, \lambda_{D}, \lambda_{F}, \lambda_{G}, \lambda_{H}, s_{1}^{+}, s_{2}^{+}, s^{-} \geq 0, \\
& \gamma_{E}^{*}=1+\frac{1}{2}\left(\frac{2}{2}+\frac{0}{4}\right)=1.5 .
\end{array}
$$

The projection of $E$ is on weak frontier

$$
\begin{aligned}
& \gamma_{D}^{*}=\operatorname{Min}\left(1+\frac{1}{2} \sum_{i=1}^{2} \frac{s_{i}^{+}}{x_{i D}}\right) \\
& \text { s.t. } 4 \lambda_{A}+7 \lambda_{B}+8 \lambda_{C}+2 \lambda_{E}+10 \lambda_{F}+12 \lambda_{G} \\
& +10 \lambda_{H}-s_{1}^{+}=2 \\
& 3 \lambda_{A}+3 \lambda_{B}+\lambda_{C}+4 \lambda_{E}+\lambda_{F}+\lambda_{G}+1.5 \lambda_{H} \\
& -s_{2}^{+}=4 \\
& \lambda_{A}+\lambda_{B}+\lambda_{C}+\lambda_{E}+\lambda_{F}+\lambda_{G}+\lambda_{H}+s^{-}=1 \\
& \lambda_{A}, \lambda_{B}, \lambda_{C}, \lambda_{E}, \lambda_{F}, \lambda_{G}, \lambda_{H}, s_{1}^{+}, s_{2}^{+}, s^{-} \geq 0 \text {, } \\
& \gamma_{D}^{*}=1+\frac{1}{2}\left(\frac{2}{4}+\frac{0}{3}\right)=1.25 \text {. }
\end{aligned}
$$

The projection is on strong frontier

$$
\begin{aligned}
& \gamma_{C}^{*}=\operatorname{Min}\left(1+\frac{1}{2} \sum_{i=1}^{2} \frac{s_{i}^{+}}{x_{i C}}\right) \\
& \text { s.t. } 4 \lambda_{A}+7 \lambda_{B}+4 \lambda_{D}+2 \lambda_{E}+10 \lambda_{F} \\
& +12 \lambda_{G}+10 \lambda_{H}-s_{1}^{+}=8 \\
& 3 \lambda_{A}+3 \lambda_{B}+2 \lambda_{D}+4 \lambda_{E}+\lambda_{F} \\
& +\lambda_{G}+1.5 \lambda_{H}-s_{2}^{+}=1 \\
& \lambda_{A}+\lambda_{B}+\lambda_{D}+\lambda_{E}+\lambda_{F}+\lambda_{G}+\lambda_{H}+s^{-}=1 \\
& \lambda_{A}, \lambda_{B}, \lambda_{D}, \lambda_{E}, \lambda_{F}, \lambda_{G}, \lambda_{H}, s_{1}^{+}, s_{2}^{+}, s^{-} \geq 0 \text {, } \\
& \gamma_{C}^{*}=1+\frac{1}{2}\left(\frac{2}{8}\right)=1.125 \text {. }
\end{aligned}
$$

The projection is on strong frontier.

In summery,

$$
\begin{aligned}
& \gamma_{C}^{*}=1.125 \\
& \gamma_{D}^{*}=1.25 \\
& \gamma_{E}^{*}=1.5 .
\end{aligned}
$$

In the case the projection of omitted $\mathrm{DMU}_{p}$ lied on weak frontier, nonzero slacks appear in optimal solution and the following approach is suggested. 


\section{Modified Nonradial Supper Efficiency Model}

First solve the following model:

$$
\begin{aligned}
& \gamma_{p}^{*}=\operatorname{Min} \frac{1+(1 / m) \sum_{i=1}^{m}\left(s_{i}^{+} / x_{i p}\right)}{1-(1 / s) \sum_{r=1}^{s}\left(s_{r}^{-} / y_{r p}\right)} \\
& \text { s.t. } \quad \sum_{j=1, j \neq p}^{n} \lambda_{j} x_{i j}-s_{i}^{+}=x_{i p} \quad i=1, \ldots, m \\
& \quad \sum_{j=1, j \neq p}^{n} \lambda_{j} y_{r j}+s_{r}^{-}=y_{r p} \quad r=1, \ldots, s \\
& \lambda_{j} \geq 0 \quad j=1, \ldots, n, j \neq p \\
& s_{i}^{+} \geq 0 \quad i=1, \ldots, m \\
& s_{r}^{-} \geq 0 \quad r=1, \ldots, s .
\end{aligned}
$$

The model, (15), can be linearized by the suggested method in [7] and solve by the simplex method.

Suppose that

$$
(\widehat{x}, \widehat{y})=\left(x_{p}+s^{+*}, y_{p}-s^{-*}\right)
$$

is the projection of $\mathrm{DMU}_{p}$ on the frontier of $\overline{P P S}$ and now solve the following model

$$
\begin{aligned}
& W_{p}^{*}=\operatorname{Max} \frac{(1 / m) \sum_{i=1}^{m}\left(\widehat{s}_{i}^{-} / \widehat{x}_{i}\right)}{(1 / s) \sum_{r=1}^{s}\left(\widehat{s}_{r}^{+} / \widehat{y}_{r}\right)} \\
& \text { s.t. } \quad \sum_{j=1, j \neq p}^{n} \lambda_{j} x_{i j}+\widehat{s}_{i}^{-}=\widehat{x}_{i} \quad i=1, \ldots, m \\
& \sum_{j=1, j \neq p}^{n} \lambda_{j} y_{r j}-\widehat{s}_{r}^{+}=\widehat{y}_{r} \quad r=1, \ldots, s \\
& \widehat{s}_{r}^{+} \geq 0 \quad r=1, \ldots, s \\
& \widehat{s}_{i}^{-} \geq 0 \quad i=1, \ldots, m, \\
& \lambda_{j} \geq 0 \quad j=1, \ldots, n, j \neq p .
\end{aligned}
$$

Set

$$
\mu_{p}^{*}=\gamma_{p}^{*}-W_{p}^{*}\left(\text { supper efficiency of } \mathrm{DMU}_{p}\right)
$$

It is evident that if $(\widehat{x}, \widehat{y})$ is strongly efficient, then $W_{p}^{*}=0$ and $\mu_{p}^{*}$ is supper efficiency score of $\mathrm{DMU}_{p}$.

\section{Modified Input Oriented Nonradial Supper Efficiency Model}

First the following model is solved:

$$
\begin{aligned}
\xi_{p}^{*}=\operatorname{Min} & \left(1+\frac{1}{m} \sum_{i=1}^{m} \frac{s_{i}^{+}}{x_{i p}}\right) \\
\text { s.t. } \quad & \sum_{j=1, j \neq p}^{n} \lambda_{j} x_{i j}-s_{i}^{+}=x_{i p} \quad i=1, \ldots, m \\
& \sum_{j=1, j \neq p}^{n} \lambda_{j} y_{r j}-s_{r}^{+\prime}=y_{r p} \quad r=1, \ldots, s \\
& s_{i}^{+} \geq 0 \quad i=1, \ldots, m \\
& s_{r}^{+\prime} \geq 0 \quad r=1, \ldots, s \\
& \lambda_{j} \geq 0 \quad j=1, \ldots, n, j \neq p .
\end{aligned}
$$

Let

$$
(\tilde{x}, \tilde{y})=\left(x_{p}+s^{+*}, y_{p}+s^{+*^{\prime}}\right) .
$$

Now solve the following problem.

$$
\begin{aligned}
W_{p}^{*}=\operatorname{Max} & \frac{(1 / m) \sum_{i=1}^{m}\left(t_{i}^{-} / \tilde{x}_{i}\right)}{(1 / s) \sum_{r=1}^{s}\left(t_{r}^{+} / \widetilde{y}_{r}\right)} \\
\text { s.t. } & \sum_{j=1, j \neq p}^{n} \lambda_{j} x_{i j}+t_{i}^{-}=\tilde{x}_{i} \quad i=1, \ldots, m \\
& \sum_{j=1, j \neq p}^{n} \lambda_{j} y_{r j}-t_{r}^{+}=\tilde{y}_{r} \quad r=1, \ldots, s \\
& t_{i}^{-} \geq 0 \quad i=1, \ldots, m \\
& t_{r}^{+} \geq 0 \quad r=1, \ldots, s \\
& \lambda_{j} \geq 0 \quad j=1, \ldots, n, j \neq p .
\end{aligned}
$$

Let

$$
\mu_{p}^{*}=\xi_{p}^{*}-W_{p}^{*}
$$

\section{Modified Output Oriented Nonradial Supper Efficiency Model}

Consider the following model:

$$
\begin{aligned}
& \eta_{p}^{*}=\operatorname{Min}\left(\frac{1}{1-(1 / s) \sum_{i=1}^{s}\left(s_{r}^{-} / y_{r p}\right)}\right) \\
& \text { s.t. } \quad \sum_{j=1, j \neq p}^{n} \lambda_{j} x_{i j}+s_{i}^{-\prime}=x_{i p} \quad i=1, \ldots, m \\
& \sum_{j=1, j \neq p}^{n} \lambda_{j} y_{r j}+s_{r}^{-}=y_{r} p \quad r=1, \ldots, s \\
& s_{i}^{-\prime} \geq 0 \quad i=1, \ldots, m \\
& s_{r}^{-} \geq 0 \quad r=1, \ldots, s \\
& \lambda_{j} \geq 0 \quad j=1, \ldots, n, j \neq p .
\end{aligned}
$$


TABLE 3

\begin{tabular}{lccccc}
\hline DMU & $C$ & $D$ & $E$ & $F$ & $G$ \\
\hline AP & 1.14 & 1.25 & 2.00 & 1.00 & 1.00 \\
SBM & 1.125 & 1.25 & 1.50 & 1.00 & 1.00 \\
Modified SBM & 1.125 & 1.25 & 1.25 & 0.90 & 0.73 \\
\hline
\end{tabular}

Let

$$
\mathrm{DMU}_{p} \text { projection }=(\bar{x}, \bar{y})=\left(x_{p}-s^{{ }^{\prime^{*}},}, y_{p}-s^{-*}\right) .
$$

Now solve the following problem:

$$
\begin{aligned}
W_{p}^{*}=\operatorname{Max} & \frac{(1 / m) \sum_{i=1}^{m}\left(\widehat{s}_{i}^{-} / \bar{x}_{i}\right)}{(1 / s) \sum_{r=1}^{s}\left(\widehat{s}_{r}^{+} / \bar{y}_{r}\right)} \\
\text { s.t. } & \sum_{j=1, j \neq p}^{n} \lambda_{j} x_{i j}+s_{i}^{-}=\bar{x}_{i} \quad i=1, \ldots, m \\
& \sum_{j=1, j \neq p}^{n} \lambda_{j} y_{r j}-s_{r}^{+}=\bar{y}_{r} \quad r=1, \ldots, s \\
& s_{i}^{-} \geq 0 \quad i=1, \ldots, m \\
& s_{r}^{+} \geq 0 \quad r=1, \ldots, s \\
& \lambda_{j} \geq 0 \quad j=1, \ldots, n, j \neq p .
\end{aligned}
$$

Let

$$
\rho_{p}^{*}=\eta_{p}^{*}-W_{p}^{*}
$$

\section{Conclusion}

In this paper, it has been shown that both AP-Model and nonradial supper efficiency model are not able to rank DMUs if the projection of omitted DMU is weak efficient in $\overline{P P S}$. The new method removes this difficulties and the example which is solved by using the new method (modified method) confirms the validity.

The results for comparing the methods are shown in Table 3.

\section{Conflict of Interests}

The authors declare that there is no conflict of interests regarding the publication of this paper.

\section{References}

[1] M. Khodabakhshi and K. Aryavash, "Ranking all units in data envelopment analysis," Applied Mathematics Letters, vol. 25, no. 12, pp. 2066-2070, 2012.

[2] M. Khodabakhshi, M. Asgharian, and G. N. Gregoriou, "An input-oriented super-efficiency measure in stochastic data envelopment analysis: evaluating chief executive officers of US public banks and thrifts," Expert Systems with Applications, vol. 37, no. 3, pp. 2092-2097, 2010.

[3] G. R. Jahanshahloo and M. Khodabakhshi, "Using input-output orientation model for determining most productive scale size in DEA," Applied Mathematics and Computation, vol. 146, no. 2-3, pp. 849-855, 2003.
[4] P. Andersen and N. C. Petersen, "A produce for ranking efficient units in data envelopment analysis," Managment Science, vol. 39, pp. 1261-1264, 1993.

[5] M. Khodabakhshi, "A super-efficiency model based on improved outputs in data envelopment analysis," Applied Mathematics and Computation, vol. 184, no. 2, pp. 695-703, 2007.

[6] M. Khodabakhshi, "An output oriented super-efficiency measure in stochastic data envelopment analysis: considering Iranian electricity distribution companies," Computers \& Industrial Engineering, vol. 58, no. 4, pp. 663-671, 2010.

[7] K. Tone, "A slacks-based measure of super-efficiency in data envelopment analysis," European Journal of Operational Research, vol. 143, no. 1, pp. 32-41, 2002.

[8] M. Khodabakhshi, "Super-efficiency in stochastic data envelopment analysis: an input relaxation approach," Journal of Computational and Applied Mathematics, vol. 235, no. 16, pp. 4576-4588, 2011.

[9] S. Ramezani-Tarkhorani, M. Khodabakhshi, S. Mehrabian, and F. Nuri-Bahmani, "On ranking decision making units withcommon weights in DEA," Applied Mathematical Modelling. In press. 


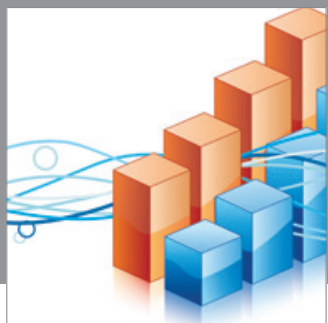

Advances in

Operations Research

mansans

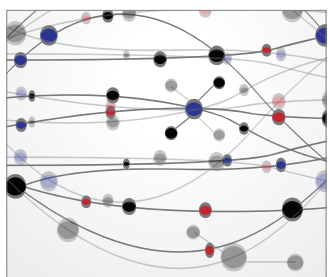

The Scientific World Journal
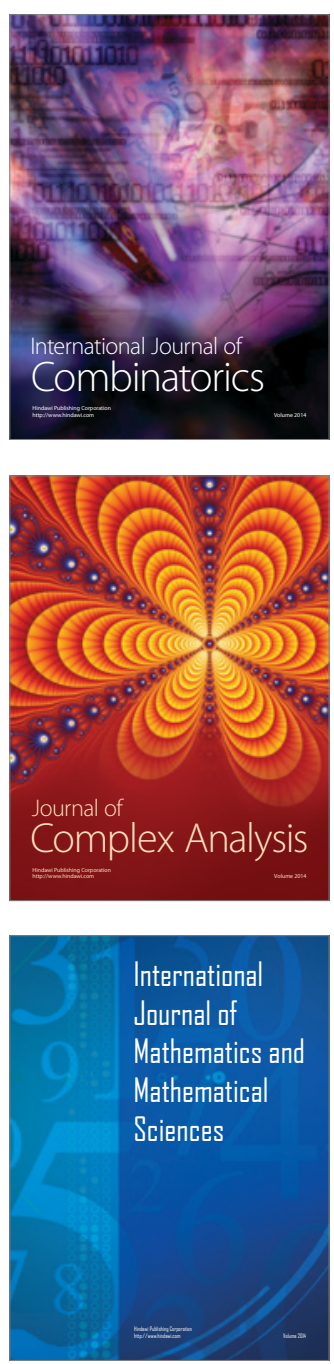
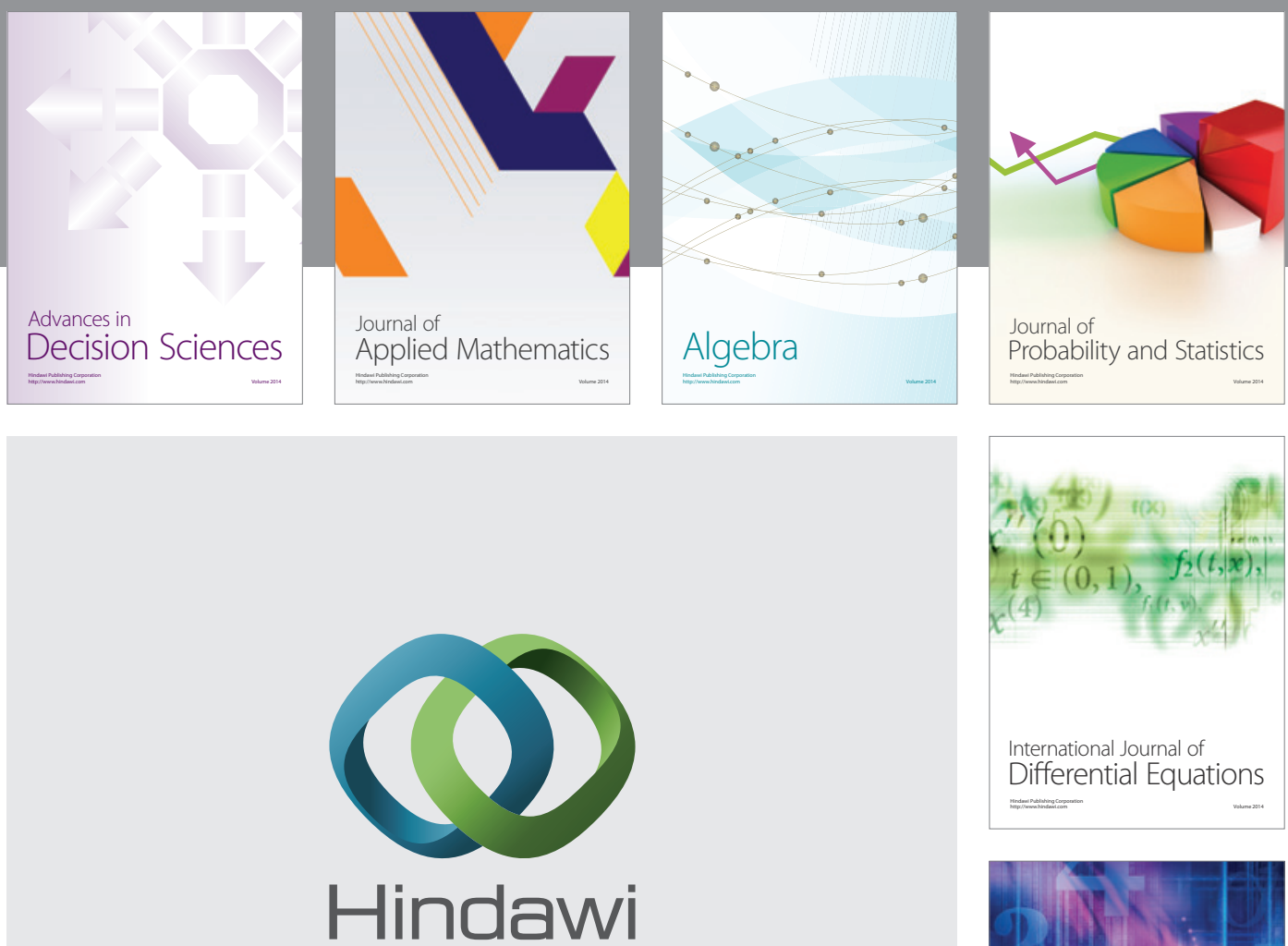

Submit your manuscripts at http://www.hindawi.com
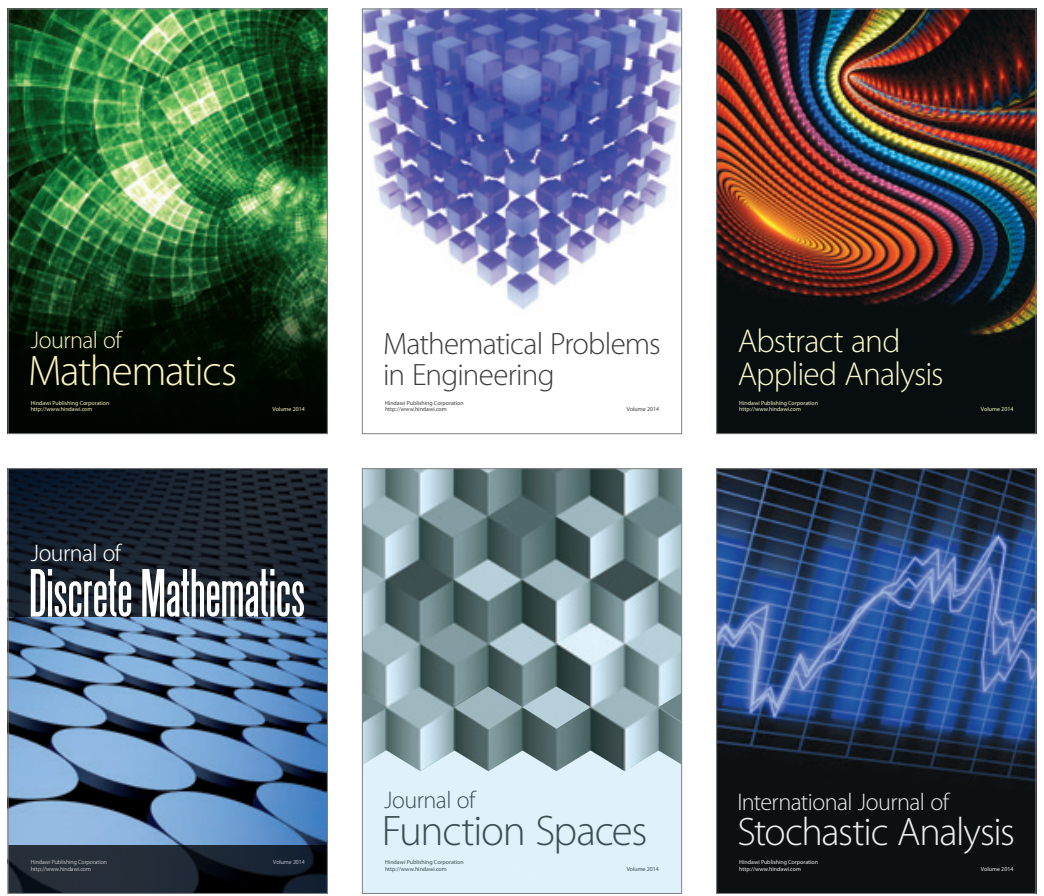

Journal of

Function Spaces

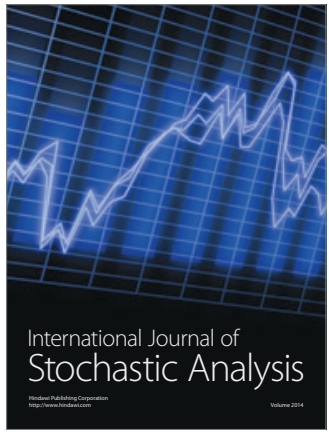

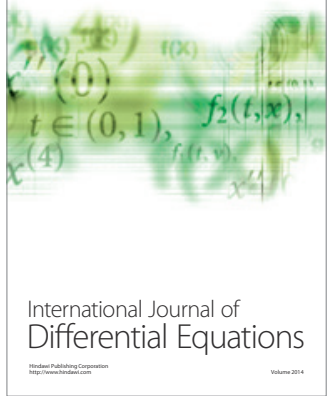
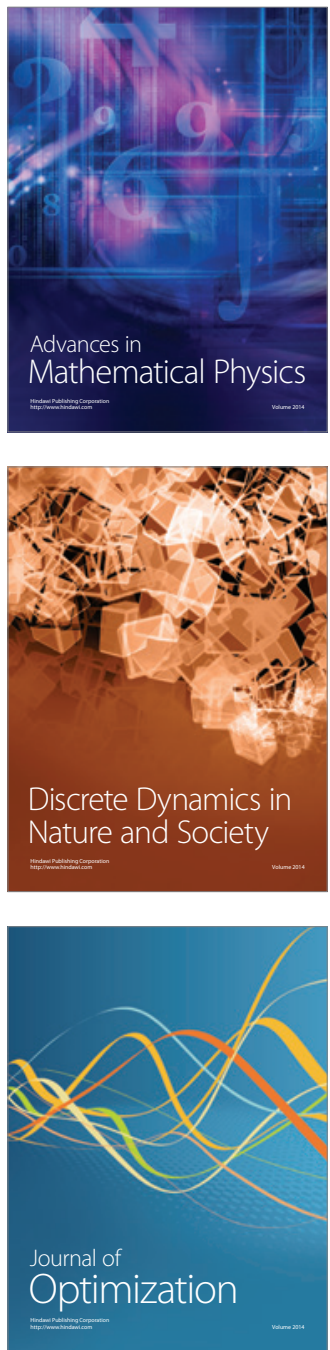